\title{
The Origin and Early Evolution of Life: Where, When and How?
}

\author{
Antonio Lazcano \\ Published online: 27 September 2012 \\ (C) Springer Science+Business Media, LLC 2012
}

\begin{abstract}
Although it is frequently taught in many schools and universities all over the world that since early antiquity philosophers and naturalists alike appealed to spontaneous generation to explain the origin of life, this was not really the case. Until the development of microscopes in the seventeenth century, spontaneous generation was seen mostly as a nonsexual reproductive mechanism of insects, maggots, and what were called "lower animals." The extraordinary descriptions of a microbial world by Anton van Leewenhoek and others opened an unsuspected dimension to the possibility of spontaneous generation, but it was not it was not until Georges Louis Leclerc de Buffon and Jean-Baptiste de Lamarck incorporated it within their transformist schemes that it was seen as the mechanism that had led to the first appearance of life on Earth.
\end{abstract}

Like his paternal grandfather and scientific predecessor Erasmus Darwin, Charles Darwin was convinced that plants and animals arose naturally from simple nonliving inorganic compounds. With very few exceptions, he carefully avoided discussing this possibility in his books. In private, however, he was much less restrained, as shown by the letter that he sent in February 1871 to Francis Hooker, in which he famously wrote, “....it is often said that all the conditions for the first production of a living organism are now present, which could ever have been present. But if (and oh what a big if) we could conceive in some warm little pond with all sorts of ammonia and phosphoric salts,-light, heat, electricity \&c. present, that a protein compound was chemically formed, ready to undergo still more complex changes, at the present day such matter $\mathrm{w}^{\mathrm{d}}$ be instantly devoured, or absorbed, which would not have been the case before living creatures were formed."

\footnotetext{
A. Lazcano $(\bowtie)$

Facultad de Ciencias, Universidad Nacional Autónoma de México, Mexico City, Mexico

e-mail: alar@ciencias.unam.mx
}

As discussed by Juli Peretó and Jesus Catalá in this issue of Evolution: Education and Outreach, Darwin's reluctance to address in public the origin of life surprised many of his friends and followers, who argued quite emphatically that Darwin's theory was incomplete until it could account for the origin of life. The list included Ernest Haeckel, whose widely read books popularized Darwin's theory and advocated that the possibility that living organisms were the evolutionary outcome of the gradual transformation of lifeless gel-like matter into protoplasm, endowed with the ability to fix atmospheric $\mathrm{CO}_{2}$, in the early atmosphere, which was a widespread notion even during Darwin's lifetime.

Equally significant, toward the end of the nineteenth century, the chemical gap separating nonliving from living matter had been bridged at least in part by the laboratory syntheses of organic molecules, which for a long time had been considered to be fundamentally different from inorganic compounds. This view soon merged with the emergent fields of biochemistry and cell biology, leading to proposals in which the origin of protoplasm was equated with the origin of life. Some of these hypotheses considered life as an emergent feature of nature and attempted to understand its origin by introducing principles of historical explanation, but most of these explanations went unnoticed, in part because they were incomplete, speculative schemes largely devoid of direct evidence and not subject to fruitful experimental testing.

This changed during the 1920s, when Alexander I. Oparin, a young Russian biochemist, proposed that life had been preceded by a lengthy period of abiotic syntheses and accumulation of organic compounds that had taken place soon after the Earth was formed. Trained both as a plant biochemist and as an evolutionary biologist, Oparin found it impossible to reconcile his Darwinian credence in a gradual, slow evolution from the simple to the complex, with the commonly held suggestion that life had emerged already endowed with an autotrophic metabolism that 
included photosynthetic pigments, enzymes, and the ability to synthesize organic compounds from $\mathrm{CO}_{2}$.

Based on the simplicity and ubiquity of fermentative reactions and on a detailed analysis of chemical synthesis and astronomical observations, Oparin attempted a theoretical reconstruction of the conditions of the primitive Earth and the evolution of organic molecules into pre-cellular systems, from which anaerobic cells that nourished themselves from the soup had evolved. Some time after, the English geneticist and polymath John B. S. Haldane independently proposed a somewhat similar scheme, suggesting that a $\mathrm{CO}_{2}$-rich atmosphere had facilitated the formation of organic compounds, assuming that viruses represented an intermediate step in the transition from the prebiotic broth to the first cells.

Oparin and Haldane sustained their proposals in part by the striking nineteenth century laboratory syntheses of biochemical compounds achieved by Wöhler, Strecker, Butlerow, and others, but the experiments of the founders of organic chemistry were not laboratory simulations of Darwins's warm little pond. The starting point of prebiotic chemistry is the 1953 Miller-Urey experiment, and the laboratory simulations that followed it soon led to the demonstration that many other monomers of biochemical significance could be readily synthesized under putative primitive conditions. As summarized here in the contribution by $\mathrm{H}$. James Cleaves, the easiness of formation under a wide variety of conditions of amino acids, purines, and pyrimidines strongly suggests that these molecules, together with urea, carboxylic acids, sugars, aliphatic and aromatic hydrocarbons, and branched and straight fatty acids, including some which are known to form bilayered membranes, were components of the prebiotic broth.

As suggested by Juan Oró in 1961, the collisions of cometary nuclei against the primitive Earth, combined with the contribution from other extraterrestrial bodies such as meteorites and interplanetary dust, may have also spiked the primitive broth with extraterrestrial organic compounds. Regardless of their ultimate origin, simple organic compounds dissolved in the primitive oceans or other bodies of water would need to be concentrated and polymerized by simple physicochemical mechanisms.

Regardless of its ultimate sources, the organic material that may have accumulated on the early Earth before life existed very likely consisted of a wide array of different types of compounds, including many of the simple compounds that play a major role in biochemistry today. However, one must be cautious, especially when teaching to students the state of the art of prebiotic chemistry. The use of terms like "primitive soup," "primordial broth," or "Darwin's warm little pond" has led in some cases to major misunderstandings, including the simplistic image of a worldwide ocean, rich in self-replicating molecules and accompanied by all sorts of biochemical monomers. The phrase "Darwin's "warm little pond," which has long been used for convenience, refers to parts of the hydrosphere where the accumulation and interaction of the products of prebiotic synthesis may have taken place. Equally important, the fact that a number of molecular components of contemporary cells can be formed nonenzymatically in the laboratory does not necessarily means that they were also essential for the origin of life or that they were available in the prebiotic environment. It is difficult to assess the complexity of the primitive soup, which very likely included organic and inorganic chemical species spiked with metallic ions, but not all the compounds or molecular structures found today in even the simplest prokaryotes. How these abiotic organic constituents were assembled into polymers and then into the first living entities is currently one the most challenging areas of research in the study of the origin of life.

As underlined by Cleaves, the remarkable coincidence between the molecular constituents of living organisms and those synthesized in prebiotic experiments is too striking to be fortuitous, and the robustness of this type of chemistry is supported by the occurrence of most of these biochemical compounds in the 4.5-billion-year-old Murchison carbonaceous chondrite and in other carbon-rich meteorites, which are indicative of the chemistry of the primitive Solar System. How life first evolved is not known, but analysis of carbonaceous chondrites and the laboratory simulations of the primitive Earth suggest that prior to the emergence of the first living systems, the prebiotic environment was endowed with: (a) a large suite of organic compounds of biochemical significance; (b) a wide array organic and inorganic catalysts, including cyanamide and other HCN derivatives, metallic ions, sulfur-rich minerals, and clays; (c) purines and pyrimidines, i.e., the potential for template-dependent polymerization reactions; and (d) membrane-forming compounds.

Indeed, catalysis, replication, and membranes are properties that we associate with living entities, as shown by discussion on metabolism, reproduction, and cell structure. The evidence summarized here suggests that the emergence of the first life forms required not the appearance of a single living molecule but the simultaneous coordination of many different components in a confluence of processes. As B. Vlaardingerbroek discusses in his paper on the sorites paradox, first stated by the post-Socratic Greek philosopher Eubulides of Miletus, this is the point of transition between the opposite ends of a continuous process. Addressing this issue in the classroom can provide a perspective for a proper recognition that life is the outcome of an evolutionary process constrained by the laws of physics and chemistry and can lead to the acceptance that many properties associated with living systems, such as replication, self-assemblage, or catalysis are also found in nonliving entities. Some systems may not be "half-alive," but they can exhibit some of the properties we associate with living entities, like selforganization, replication, or Darwinian evolution. 
As noted by Purifcación Lopez-Garcia and David Moreira in their article on viruses included in this issue, there is a venerable intellectual tradition based on the apparent simplicity and the replicative abilities of viruses that links them to the origin of life. However, there is no evidence that viruses are indeed primitive. Such hypothesis keep resurfacing from time to time, including recent proposals that the earliest cells were preceded by stages of evolution involving virus-like genetic polymers of abiotic origin synthesized within iron-sulfide mineral compartments in warm hydrothermal environments, which are assumed to be ancestral to extant RNA viruses, retroviruses, and later on DNA viruses.

It is of course possible that (some) RNA viral genomes (or parts of them) originated during the RNA/protein world that preceded the evolutionary development of DNA biosynthesis and the divergence of the three major cell lineages addressed by Arturo Becerra and Luis Delaye in this issue. However, the proposal that such stages took place within the boundaries of hydrothermal systems is not supported by current descriptions of the primitive environment and begs major chemical issues associated with the abiotic synthesis, accumulation, and stability of ribonucleotides and polyribonucleotides.

The surprising ability of RNA molecules to catalyze an increasingly large number of chemical reactions has lent strong support to the possibility of the so-called RNA world, and as discussed here with surprising clarity by Andrew Ellington, greatly simplifies the understanding of the origin of protein biosynthesis and of the genetic code. The RNA world hypothesis, however, does not imply that life should be stripped of its identity and reduced to a mere collection of autocatalytic RNA molecules. There are many definitions of the RNA world, but they do not imply that ribozymes suddenly appeared on the primitive Earth endowed with the miraculous ability to construct a fully functional living being.

The chemical instability of RNA and the problems associated with the abiotic synthesis of its monomers have led some to suggest that RNA itself may have been preceded by simpler genetic polymers, whose appearance marked the beginning of true heredity and hence of natural selection. Not all agree with this possibility, but in any case, at the time being, the hiatus between the primitive soup and the RNA world is discouragingly enormous.

The stunning widening of the catalytic repertoire of RNA under in vitro conditions that allow the evolution of new chemical abilities to appear under selection pressures and to catalyze an increasingly large number of reactions has lent strong support to the possibility of the RNA world. Unfortunately, it is not possible to assign a precise chronology to its emergence or to other events that may have preceded extant DNA/RNA/proteins cells. The recognition that life is a very ancient phenomenon runs parallel to the limits imposed by a scarce Archaean geological record that becomes increasingly blurred as we go back in time, with very few rocks older than 3.5 billion years old.

As argued by Becerra and Delaye, a comparative genomics provides important clues on very early stages of biological evolution, which can be easily implemented in the classroom. It is true that the applicability of this approach cannot be extended beyond a threshold that corresponds to a period of cellular evolution in which ribosome-mediated protein synthesis was already in operation, but as argued by Renato Fani, bioinformatics tools, combined with a huge amount of information on classical microbial genetics, allow significant insights into the origin and early evolution of metabolic pathways. Unfortunately, it is neither possible to assign a precise chronology to the origin and earliest evolution of cells nor can we obtain direct evidence on older stages, which may have included ribozyme-mediated biosynthetic pathways.

It is unlikely that the paleontological record will provide direct data on how life first appeared and evolved. There is neither geological evidence of the environmental conditions on the Earth at the time of the origin of life nor any fossil register of the evolutionary processes that preceded the appearance of the first cells. Direct information is lacking not only on the composition of the terrestrial atmosphere during the period of the origin of life but also on the temperature, ocean $\mathrm{pH}$ values, and other general and local environmental conditions which may or may not have been important for the emergence of life. The attributes of the first living organisms are also unknown. They were not like extant microbes, but probably simpler than any cell now alive, and may have lacked not only proteinbased catalysis, but also perhaps even the familiar genetic macromolecules, with their ribose-phosphate backbones.

The study of the origin of life is a legitimate scientific question and an alluring intellectual endeavor. Those studying it know they have plenty to be modest about, and they tend to be. We will never know in full detail how life first appeared. If this sounds pessimistic, it is; there are, after all, many gaps in the papers included in this issue of Evolution: Education and Outreach. However, we do not need, as argued by the highly vocal creationist movements that have reappeared in the U.S. and are expanding to other countries, to introduce supernatural explanations based on so-called intelligent design. The study of the origin of life has been transformed from a purely speculative discussion into a workable research program. It is true that it is ridden with controversies, but such disagreements are recognized by the scientific community as intellectual challenges and have in most cases led to fruitful clarifying debates. Evidence of scientific ignorance is not evidence for creation. There are manifold historical records that allow us to reconstruct, with different degrees of precision, the evolutionary processes that preceded the emergence of life, and the mere fact that we can address this problem is, in itself, a major intellectual achievement that can be conveyed to students and teacher alike. 